\title{
MODELAGEM CAIXA PRETA DO TANQUE DE AQUECIMENTO DA PLANTA DIDÁTICA SMAR PD3: UMA ATUALIZAÇÃ̃O
}

\section{BLACK BOX MODELING OF THE HEATING TANK OF THE SMAR PD3 DIDATIC PLANT: AN UPDATE}

\section{J. M. CALLEGARI ${ }^{1}$, A. G. TÔRRES ${ }^{2}$, J. V. NICACIO ${ }^{3}$ e L. B. FELIX ${ }^{2 *}$}

${ }^{1}$ Programa de Pós-Graduação em Engenharia Elétrica, Centro Federal de Educação Tecnológica de Minas Gerais, Belo Horizonte, Minas Gerais, Brasil

${ }^{2}$ Universidade Federal de Viçosa, Departamento de Engenharia Elétrica, Viçosa, Minas Gerais, Brasil

${ }^{3}$ Universidade Federal de Viçosa, Departamento de Engenharia Agrícola, Viçosa, Minas Gerais, Brasil

${ }^{*}$ Leonardo Bonato Felix. Universidade Federal de Viçosa, Departamento de Engenharia Elétrica, Viçosa, Minas Gerais, Brasil, Telefone: +55 (31) $3612-6400$. Email: leobonato@ufv.br (L.B.FELIX).

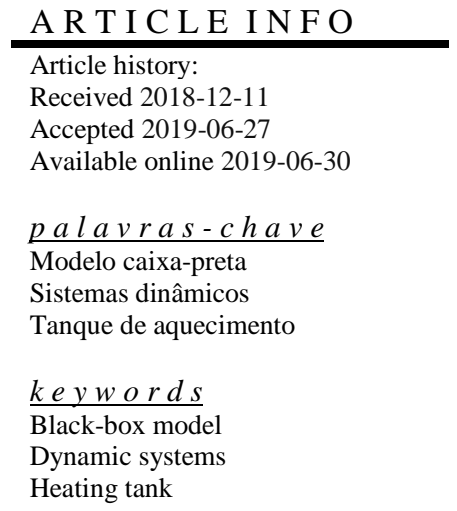

\section{R E S U M O}

Este trabalho propõe a modelagem caixa-preta do tanque de aquecimento da planta SMAR PD3 (sistema) e o compara com o modelo teórico baseado na física do processo. O estudo se dá com a flutuação da temperatura da água no tanque (saída), de acordo com degraus de potência e vazão (entradas) aplicados no sistema. Os resultados apontam uma melhor performance dos modelos Box-Jenkins em relação aos demais, sob a ótica de dois critérios: análise de resíduos e do coeficiente de determinação. O modelo escolhido e o modelo baseado na física do processo apresentaram coeficiente de determinação médio de aproximadamente $90 \%$ e $80 \%$, respectivamente, considerando 7 casos para validação. Além disso, o modelo proposto apresentou melhor desempenho estatisticamente significativo em todos os casos quando comparado com o modelo teórico.

\section{A B S T R A C T}

This work proposes the black box modeling of the heating tank of the SMAR PD3 plant (system) and compares it with the theoretical model based on process physics. The study considers the water temperature fluctuation in the tank (output), according to power and flow (inputs) steps applied in the system. The results show a better performance of the Box-Jenkins models than the others, from the standpoint of two criteria: residue analysis and determination coefficient. The black-box model and a previous model based on the process physics obtained an average determination coefficient of approximately $90 \%$ and $80 \%$, respectively, considering 7 cases for validation. In addition, the proposed model presented better statistically significant performance in all cases when compared to the theoretical model. 


\section{INTRODUÇ̃̃̃O}

Entender e explicar fenômenos observados na natureza tem sido a grande motivação para o ser humano desenvolver ferramentas capaz de auxiliá-lo (AGUIRRE, 2007). É neste contexto que entram os modelos matemáticos: análogo matemático que representa algumas características observadas de um sistema analisado (JOHANN; PEREIRA; SILVA, 2015). Em termos práticos, os modelos matemáticos podem descrever o comportamento de certos fenômenos analisados (sistemas) e é uma representação aproximada de apenas algumas características do sistema real. Estas características dependem do objetivo para o qual o modelo está sendo desenvolvido.

Há três categorias de técnicas de modelagem: (i) Modelagem caixa branca; (ii) Modelagem caixa preta; (iii) Modelagem caixa cinza. Para a modelagem caixa branca é preciso conhecer bem o sistema, assim como as leis físicas que o descrevem. Infelizmente, o tempo e conhecimento necessário para este tipo de modelagem partindo do equacionamento dos fenômenos envolvidos nem sempre é viável (CORRÊA; AGUIRRE, 2004). Na modelagem caixa preta, exige-se pouco ou nenhum conhecimento prévio do sistema, baseando-se apenas na observação da relação de causa e efeito entre variáveis de entrada e saída. Em outras palavras, nenhuma informação sobre o sistema está disponível além daquelas fornecidas pelos dados ou, se disponível, não é usada na obtenção do modelo. Finalmente, a modelagem caixa cinza inclui as características das outras abordagens, ou seja, qualquer procedimento que não se enquadra nas duas classificações anteriores pode ser denominado de modelagem caixa cinza.

Para descrever a dinâmica de sistemas, os modelos caixa preta são utilizados neste trabalho. Para tal, são necessários técnicas de álgebra linear, conceitos de estatística e procedimentos de otimização sem restrições para determinação de seus parâmetros.

Em um ambiente industrial, por exemplo, é comum malhas para controle dos processos produtivos. No entanto, para se obter sucesso no controle de um sistema é necessário o estudo da sua dinâmica sem auxílio ou interferência dessas malhas (MORAES et al., 2017). Em geral, os sistemas reais são dinâmicos, isto é, descritos por equações diferenciais ou equações a diferenças (KLUEVER, 2017). Assim, o entendimento da resposta transitória de sistemas de diversas ordens é possível variando as entradas e analisando a resposta obtida. As entradas devem ser variadas visando obter dados dinâmicos com a maior quantidade de informação do sistema que se deseja modelar. O sucesso dos modelos caixa-preta depende fortemente da qualidade desses dados.

A modelagem prévia do sistema facilita a definição da melhor estratégia a ser adotada (DOMINGOS et al., 2017). Um modelo matemático pode auxiliar, muitas vezes, nas tomadas de decisões e definições dos limites de operação do sistema, já que há sempre o interesse em otimizar a produção e reduzir custos de manutenção, reparo e troca de equipamentos. Desta forma, a motivação deste trabalho baseia-se na obtenção de um modelo apurado e validado, que permite:

- Realizar simulações utilizando-o sob condições físicas extremas. Através da resposta obtida pelo modelo matemático, é possível dimensionar proteções para que a planta não opere nestas condições e não sofra danificações;

- Obter resultados da dinâmica da planta por meio do modelo matemático, sem a necessidade de interromper a produção do sistema, evitando decréscimo de lucro;

- Estimar os ganhos dos controladores baseados no modelo matemático da planta, economizando horas de sintonia empírica.

DOMINGOS et al. (2017) utiliza da física do processo para desenvolver um modelo capaz de explicar a dinâmica do tanque de aquecimento da planta SMAR PD3. O objetivo deste trabalho é propor uma atualização do modelo caixa branca, utilizando a modelagem caixa-preta. Os resultados do modelo proposto são comparados com o modelo de DOMINGOS et al. (2017), considerando 7 diferentes conjuntos de dados de validação. O banco de dados foi obtido em laboratório e constitui-se dos degraus de potência [W] e vazão $\left[\mathrm{Lh}^{-1}\right]$, assim como a resposta de temperatura $\left[{ }^{\circ} \mathrm{C}\right]$ da água no tanque de aquecimento. O desempenho dos modelos é simulado em condições de operação da qual se tenha medições para validação.

Este artigo está organizado da seguinte forma: Seção 2 fornece os conceitos preliminares, apresentando diversos modelos discretos e contínuos. Seção 3 introduz as medidas de desempenho para análise da qualidade dos modelos, assim como a planta sob estudo e a metodologia adotada para comparação dos modelos. Seção 4 apresenta os resultados e discussão. Finalmente, as conclusões estão dispostas na Seção 5.

\section{CONCEITOS PRELIMINARES}

\subsection{Modelos discretos}

Modelos discretos são muito utilizados na identificação de sistemas. Um modelo geral pode ser definido por (AGUIRRE, 2007):

$$
A(z) y(k)=\frac{B(z)}{F(z)} u(k)+\frac{C(z)}{D(z)} v(k),
$$

onde $\mathrm{y}(\mathrm{k})$ e $\mathrm{u}(\mathrm{k})$ são a saída e a entrada do sistema, respectivamente. $v(k)$ é o ruído branco. $\mathrm{A}(\mathrm{z}), \mathrm{B}(\mathrm{z}), \mathrm{C}(\mathrm{z}), \mathrm{D}(\mathrm{z})$ e $\mathrm{F}(\mathrm{z})$ são polinômios genéricos de ordem $n$, dados por:

$$
\begin{aligned}
& A(z)=1-a_{1} z^{-1}-\cdots-a_{n_{y}} z^{-n_{y},} \\
& B(z)=b_{1} z^{-1}+\cdots+b_{n_{u}} z^{-n_{u},} \\
& C(z)=1+c_{1} z^{-1}+\cdots+c_{n_{v}} z^{-n_{v},} \\
& D(z)=1+d_{1} z^{-1}+\cdots+d_{n_{d}} z^{-n_{d},} \\
& F(z)=1+f_{1} z^{-1}+\cdots+f_{n_{f}} z^{-n_{f},}
\end{aligned}
$$

onde $\mathrm{z}^{-1}$ é o operador de atraso, isto é, $\mathrm{y}(\mathrm{k}) \mathrm{z}^{-1}=\mathrm{y}(\mathrm{k}-1)$. Através do modelo geral, dado na Equação 1, define-se alguns modelos discretos: (i) Auto-regressivo com entradas exógenas externas (ARX); (ii) Box-Jenkins (BJ); (iii) Output error (OE).

O modelo ARX pode ser obtido fazendo $\mathrm{C}(\mathrm{z})=\mathrm{D}(\mathrm{z})=$ $\mathrm{F}(\mathrm{z})=1$, conforme apresentado na Equação 7: 


$$
y(k)=\frac{B(z)}{A(z)} u(k)+\frac{v(k)}{A(z)} .
$$

A representação em diagramas de bloco é dada na Figura 1 (a). O ruído branco é aplicado diretamente à dinâmica do sistema. Já o ruído $\mathrm{e}(\mathrm{k})=\mathrm{v}(\mathrm{k}) / \mathrm{A}(\mathrm{z})$ adicionado à saída, é modelado como um processo branco filtrado por um filtro autoregressivo, com polos idênticos aos do processo estudado (raízes do polinômio A(q)) (AGUIRRE, 2007).

O modelo de BJ pode ser obtido a partir da Equação 1, considerando $\mathrm{A}(\mathrm{z})=1$ :

$$
y(k)=\frac{B(z)}{F(z)} u(k)+\frac{C(z)}{D(z)} v(k) .
$$

É um modelo mais genérico em relação ao anterior, onde se consideram funções de transferências independentes para o ruído $\mathrm{C}(\mathrm{z}) / \mathrm{D}(\mathrm{z})$ e para a entrada, $\mathrm{B}(\mathrm{z}) / \mathrm{F}(\mathrm{z})$ (WERNER; RIBEIRO, 2003; MARQUES, 2012). A representação em diagramas de bloco da Equação 8 é apresentado na Figura 1 (b).

Pode-se enumerar três etapas para construção do modelo BJ: (i) Identificação; (ii) Estimação e (iii) Verificação. Inicialmente, estima-se os parâmetros do modelo de acordo com os dados coletados; a verificação é feita através da predição de valores futuros da série (WERNER; RIBEIRO, 2003; MARQUES, 2012). Caso o modelo não seja adequado, volta-se à fase de identificação.

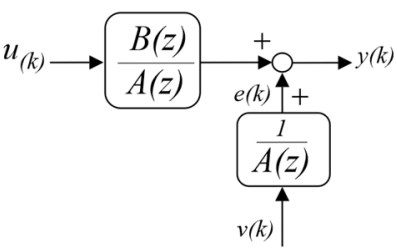

(a)

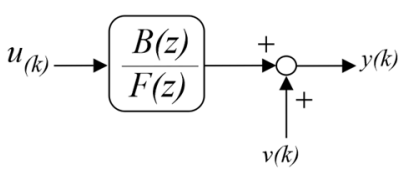

(c)

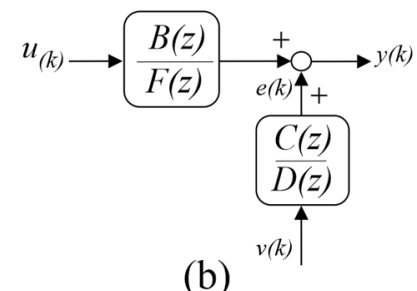

(b)

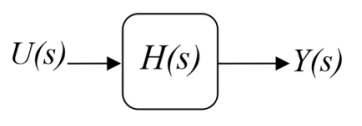

(d)
Figura 1 - Diagrama de blocos para o modelo (a) ARX. (b) BJ. (c) OE. (d) FT. Adaptado de (AGUIRRE, 2007).

Por fim, o modelo OE pode ser obtido a partir do modelo geral com $\mathrm{A}(\mathrm{z})=\mathrm{D}(\mathrm{z})=\mathrm{C}(\mathrm{z})=1$ :

$$
y(k)=\frac{B(z)}{F(z)} u(k)+v(k),
$$

onde o ruído aleatório $\mathrm{v}(\mathrm{k})$ é diretamente adicionado à saída do modelo. A Figura 1 (c) apresenta o diagrama de blocos da Equação 9.

\subsection{Modelos contínuos}

A função de transferência contínua (FT) traduz o comportamento dinâmico de um par entrada-saída de um sistema. É obtida pela transformada de Laplace da resposta ao impulso (ARRUDA; BARROS, 2003). A FT H(s) da resposta ao impulso $h(t)$ de um sistema pode ser de ordem $N, \operatorname{com} N \geq$ 1. Na maioria dos processos, é possível aproximar H(s) para uma FT de primeira ordem e, em alguns casos, de segunda ordem. Matematicamente, pode-se escrever H(s) em termos da entrada U(s) e saída $Y(s)$ :

$$
Y(s)=H(s) U(s) .
$$

A Figura 1 (d) apresenta a representação em diagramas de bloco da Equação 10.

\section{METODOLOGIA}

A ferramenta MATLAB $^{\circledR}$ e o aplicativo System Identification Toolbox são utilizados para desenvolvimento dos modelos.

\subsection{Medidas de desempenho: predições um passo à frente e} livre

Um bom modelo reproduz os dados observados e deve ser validado a partir de um conjunto de dados diferente daquele utilizado para modelagem. Desta forma, deseja-se saber se o modelo consegue explicar outro conjunto de dados de um mesmo sistema.

A predição um passo à frente consiste em predizer o valor seguinte, utilizando a massa de dados. Para tal, toma-se observações dos dados até o instante $k$ para predizer a saída no instante $k+1$ (COUTINHO; SILVA; DELGADO, 2016). A predição no instante $k+1$ não é utilizada para predizer o instante $k+2$ e, portanto, o erro é sempre o menor possível. Fica evidente que este tipo de predição não é um bom parâmetro para validação de modelos (AGUIRRE, 2007).

Outra forma de verificar um modelo é utilizar predições passadas para compor o vetor de regressores a fim de continuar fazendo a predição. Este tipo de simulação é chamado predição livre ou de infinitos passos à frente (AGUIRRE, 2007). Trata-se de um teste mais refinado para conclusões sobre a qualidade do modelo.

Todas as análises de qualidade dos modelos são feitas utilizando um banco de dados de validação, diferente daquele utilizado para a modelagem.

\subsection{Medidas de desempenho: $\mathrm{R}^{2}$ e RMSE}

A raiz do erro quadrado médio (RMSE) é calculado por:

$$
R M S E=\frac{\sqrt{\sum_{k=1}^{N}(y(k)-\hat{y}(k))^{2}}}{\sqrt{\sum_{k=1}^{N}(y(k)-\bar{y})^{2}}},
$$

onde $\hat{y}(k)$ é a saída do modelo e $\bar{y}$ é a média temporal do sinal de saída medido $\mathrm{y}(\mathrm{k})$.

$\mathrm{O}$ coeficiente de determinação $\mathrm{R}^{2}$ é um índice estatístico utilizado para medir o ajuste de um modelo linear; é um bom parâmetro a ser considerado na escolha do modelo mais adequado. A Equação 12 apresenta o cálculo deste parâmetro:

$$
R^{2}=(1-R M S E) 100 \%
$$

Quanto maior o valor de $\mathrm{R}^{2}$ (de 0 a $100 \%$ ), melhor o modelo se ajusta ao conjunto de dados medidos.

\subsection{Tanque de aquecimento da planta SMAR PD3}

A planta SMAR PD3 tem o objetivo de demonstrar 
didaticamente a operação das diversas malhas de controle utilizando equipamentos e ferramentas de configuração similares àqueles aplicadas em controle industrial (SMAR, 2012). A planta foi desenvolvida com o objetivo de ser manipulada, sendo acessível devido ao seu arranjo compacto. As características e situações observadas em contextos industriais são traduzidas nas malhas implementadas, levando ao ambiente acadêmico situações similares às dispostas nas empresas.

A Figura 2 (a) apresenta a vista frontal da planta didática SMAR PD3. Trata-se de uma planta composta por tanques, transdutores, bombas, atuadores, painel de operação e um Controlador Lógico Programável (CLP) modular da SMAR (GOMES; NICACIO; TÔRRES, 2017; OLIVEIRA et a., 2012).

Inúmeras configurações de operação podem ser ajustadas devido sua flexibilidade. A circulação de líquidos entre os tanques, medição de variáveis analógicas e controle implementado via software no CLP, por meio da plataforma SYSTEM302, são algumas possibilidades de simulação na planta didática (SANTOS, 2016, ANDRADE; ARAÚJO, 2013). Apesar de ser composta por vários processos, este trabalho deu enfoque na modelagem do tanque de aquecimento, indicado pelo número 20 na Figura 2 (a). Este processo pode ser resumido nas seguintes etapas:

- A água é inicialmente armazenada no tanque de abastecimento (22);

- A água é bombeada pela bomba 1 (16) para o tanque de aquecimento (20);

- As resistências R1 e R2 (18) aquecem a água no tanque 20, onde a vazão de entrada neste tanque também pode ser ajustada;

- A válvula (1) é responsável pela vazão de água de entrada no tanque;

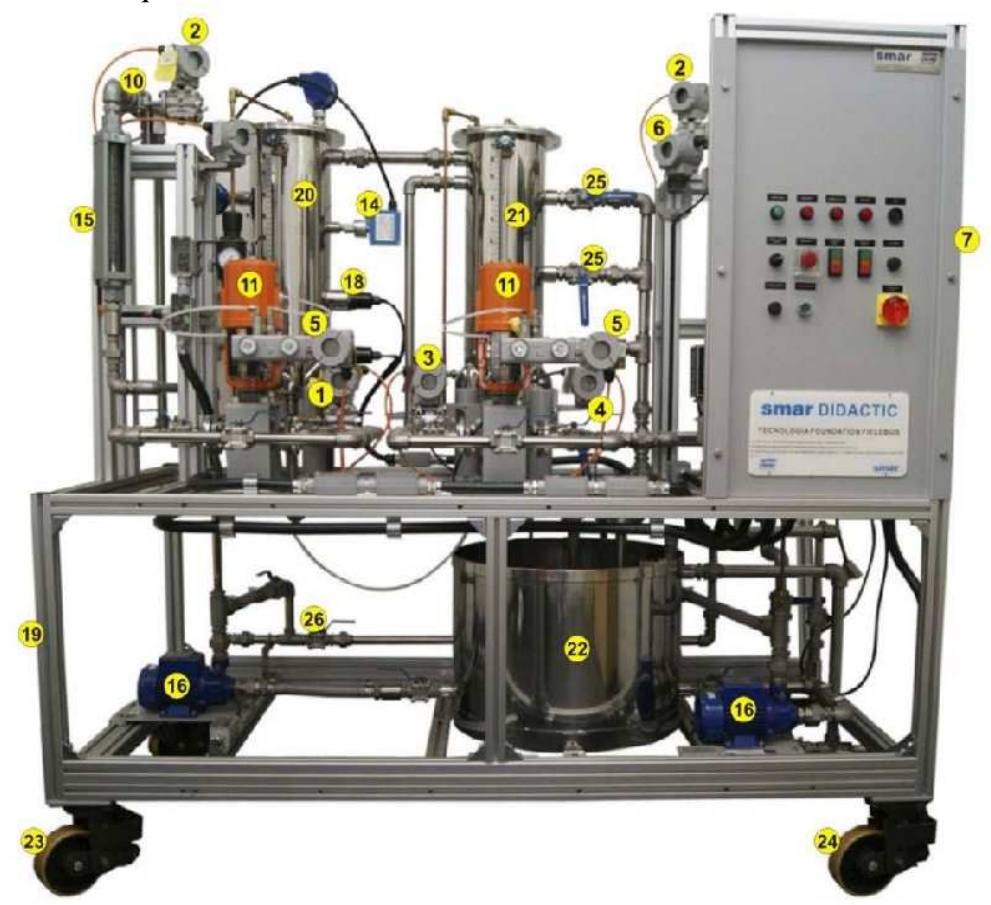

(a)
- O rotâmetro (15) mede a vazão instantânea de água de entrada no tanque de aquecimento 20 ;

- A água quente que deixa o tanque de aquecimento é descartada após passar pelo tanque de mistura (21).

Trata-se de um sistema com duas entradas: (i) potência dissipada nos resistores; e (ii) vazão de água. Além disso, apresenta uma única saída: medição da temperatura da água. Alguns sensores presentes na própria planta didática permitem a coleta dos dados das entradas e da respectiva saída. A Figura 2 (b) apresenta o diagrama P\&I da planta em estudo, de acordo com a normatização ISA 5.1 .

Para medição de temperatura no tanque de água quente, um sensor PT-100 (ou termorresistência) foi utilizado. A atuação direta na variação da vazão de entrada é feita por uma válvula tipo globo, acionada de forma pneumática. $\mathrm{O}$ conversor estático de potência é utilizado para alimentar as resistências elétricas responsáveis por aquecer a água: Um sinal de 4-20 mA é aplicado na entrada do conversor, o que corresponde à uma potência de 0-8 kW dissipada no resistor.

O rotâmetro (FI) de água indica o valor instantâneo da vazão de água no respectivo circuito, enquanto a bomba hidráulica na planta didática promove a circulação de água pelas tubulações e no tanque (SMAR, 2012).

Os equipamentos que realizam a interface com a rede Foundation Fieldbus (FF) e os sensores/atuadores são os transmissores de temperatura (TIT31), posicionadores de válvulas (FY31), transmissor de pressão diferencial para medição de vazão (FIT31) e conversor 4-20 mA (TY31). Este último converte a informação que chega da rede FF para uma saída 4-20 mA, que é aplicado no conversor de potência. Detalhes individuais de cada equipamento são encontrados em (SMAR, 2012; SANTOS, 2016).

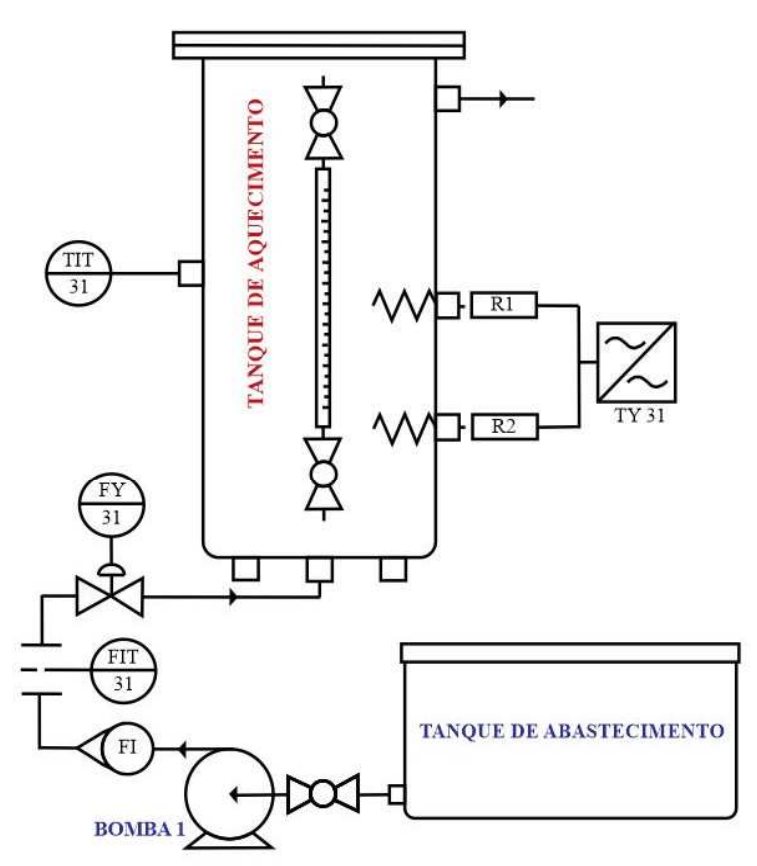

(b)

Figura 2 - (a) Vista frontal da planta (SMAR, 2012). (b) Diagrama P\&I do tanque de aquecimento da planta didática. 


\subsection{Modelagem caixa branca de MORAES et al. (2017).}

A resposta dinâmica de tanques que possuem a capacidade de armazenar líquidos ou gases pode ser aproximada por um sistema de primeira ordem. MORAES et al. (2017) realizou a modelagem caixa branca da planta da Seção 3.3, considerando sua dinâmica como uma FT de primeira ordem, obtendo o seguinte resultado:

$$
G(s)=\frac{T(s)}{P(s)}=\frac{1}{A h \rho C_{p} s+\rho C_{p} Q} .
$$

A modelagem foi feita considerando a vazão $Q$ como um parâmetro constante. Assim, várias funções de transferência foram obtidas para o modelo, uma para cada valor de $Q$. A Tabela 1 apresenta os parâmetros da Equação 13.

Tabela 1 - Parâmetros físicos da planta.

\begin{tabular}{l|ll} 
Parâmetros & Símbolo & Valor \\
Área base do reservatório & $\mathrm{A}$ & $0,034241349 \mathrm{~m}^{2}$ \\
Densidade da água & $\rho$ & $1000 \mathrm{~kg} / \mathrm{m}^{3}$ \\
Altura do reservatório & $\mathrm{h}$ & $0,7 \mathrm{~m}$ \\
Capacidade calorífica & $\mathrm{C}_{\mathrm{p}}$ & $4186 \mathrm{~J} / \mathrm{kg}^{\circ} \mathrm{C}$ \\
Vazão volumétrica & $\mathrm{Q}$ & $\mathrm{x} \mathrm{m} / \mathrm{s}$ \\
\hline
\end{tabular}

\subsection{Dados de modelagem e validação}

A Figura 3 apresenta o fluxograma utilizado no processo de identificação e validação dos modelos propostos. Para modelagem, escolheu-se, estrategicamente, dados de entradas (sinal do conversor e vazão de entrada) aplicadas em tempos diferentes, isto é, pulso de potência aplicado no instante $34 \mathrm{~s}$ e retirado em 2916 s e degrau de vazão aplicado no instante 1970 s. Desta forma, estes dados de entrada e a respectiva saída contêm maiores informações da dinâmica do sistema.

Além disso, o conjunto de dados de modelagem consiste em 3250 pontos amostrados a cada $1 \mathrm{~s}$ para cada variável medida, durante ensaios realizados na planta SMAR PD3 por quase $54 \mathrm{~min}$.
A partir dos dados de modelagem, foram obtidos diferentes modelos no que diz a respeito aos parâmetros e à ordem, conforme descrito na Seção 2. Estes modelos foram testados através de outros conjuntos de dados (denominados dados de validação), por meio da análise de resíduos, $\mathrm{R}^{2} \mathrm{e}$ RMSE.

Foram escolhidos $\mathrm{N}=7$ diferentes conjuntos de validação (ou Casos), conforme apresentado na Tabela 2. Os degraus de entrada, seja de potência seja de vazão, variam de um conjunto para outro, assim como a duração do ensaio. Por exemplo, o sistema é excitado com $8000 \mathrm{~W}$ de potência e 200 $\mathrm{L} / \mathrm{h}$ no Caso 1 . No Caso 5 é excitado com $2000 \mathrm{~W}$ e $600 \mathrm{~L} / \mathrm{h}$, enquanto nos Casos 6 e 7, aplica-se $8000 \mathrm{~W}$ e $4000 \mathrm{~W}$ para uma vazão de $600 \mathrm{~L} / \mathrm{h}$, respectivamente.

Tabela 2 - Resumo dos dados de validação.

\begin{tabular}{lllll}
$\begin{array}{c}\text { Dados de } \\
\text { Validação }\end{array}$ & $\begin{array}{c}\text { Duração } \\
\text { do Ensaio } \\
\text { (s) }\end{array}$ & $\begin{array}{c}\text { Tempo de } \\
\text { Amostragem } \\
(\mathbf{s})\end{array}$ & $\begin{array}{c}\text { Entradas } \\
\text { Pot. } \\
(\mathbf{k W})\end{array}$ & $\begin{array}{c}\text { Vazão } \\
\left(\mathbf{L h}^{-1}\right)\end{array}$ \\
\hline Caso 1 & 1688 & 1 & 8 & 200 \\
Caso 2 & 2230 & 1 & 2 & 200 \\
Caso 3 & 1507 & 1 & 4 & 200 \\
Caso 4 & 1505 & 1 & 8 & 400 \\
Caso 5 & 1002 & 1 & 2 & 600 \\
Caso 6 & 1211 & 1 & 4 & 600 \\
Caso 7 & 912 & 1 & 8 & 600 \\
\hline
\end{tabular}

\subsection{Análise de resíduos}

Define-se o vetor de resíduos $\xi$ como:

$\xi=y-\hat{y}$,

onde y e $\hat{y}$ são, respectivamente, a saída do conjunto de validação e saída estimada pelo modelo. A variância dos resíduos não é um indicador para validar modelos, e sim para verificar se o modelo está polarizado (tendencioso) na determinação de seus parâmetros.

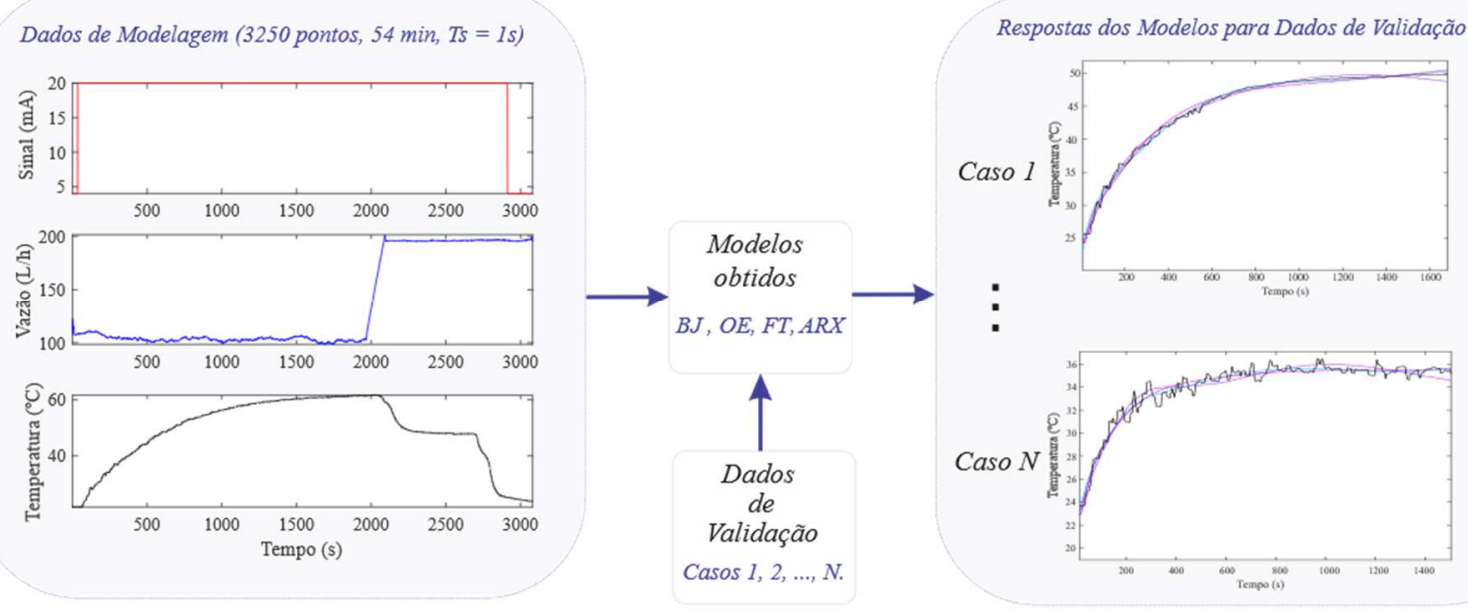

Figura 3 - Diagrama do processo de modelagem e validação dos modelos. 
A análise de resíduos objetiva verificar se os resíduos da Equação 14 são ou não aleatórios. Se o vetor de resíduos for uma variável aleatória branca, então não há informações úteis nos resíduos (AGUIRRE, 2007) e o modelo explicou tudo o que era explicável nos dados. Em termos matemáticos, a autocorrelação do resíduo deve ser igual a um impulso na amostra 0 e a correlação cruzada entre entrada e resíduo deve ser 0 . Assim,

$$
\begin{aligned}
& r_{\xi \xi}=\operatorname{corr}(\xi, \xi)=\delta(0), \\
& r_{u \xi}=\operatorname{corr}\left(u_{i}, \xi\right)=0, \forall \text { re } i=\{1,2\},
\end{aligned}
$$

onde os subíndices 1 e 2 representam as entradas 4-20 mA e a vazão, respectivamente. Estatisticamente, considera-se 0 os valores dentro de um intervalo aceitável.

Foi definido, como critério de seleção, que a resposta dos modelos deve satisfazer simultaneamente as Equações 15 e 16 (AGUIRRE, 2007) e apresentar um coeficiente de determinação médio acima de $80 \%$ para os casos analisados, i.e., uma forte correlação.

\section{RESULTADOS E DISCUSSÃO}

A partir dos dados de modelagem foram selecionados 18 modelos, sendo 7 modelos BJ, 6 modelos OE, 3 modelos FT e 2 modelos ARX.

A Tabela 3 apresenta o $\mathrm{R}^{2}$ dos modelos propostos para os 7 casos de validação da Tabela 2. Os modelos ARX apresentaram baixo $\mathrm{R}^{2}$ médio nas predições livres, na ordem de $10 \%$. Portanto, foram desconsiderados.

Tabela 3 - Coeficiente de determinação de cada modelo para os Casos 1 ao 7.

\begin{tabular}{llllllll} 
Modelos & & \multicolumn{7}{c}{$\mathbf{R}^{\mathbf{2}}(\mathbf{\%}) \mathbf{- C a s o s}$} \\
& $\mathbf{1}$ & $\mathbf{2}$ & $\mathbf{3}$ & $\mathbf{4}$ & $\mathbf{5}$ & $\mathbf{6}$ & $\mathbf{7}$ \\
\hline bj_1 & $\mathbf{9 5 , 3}$ & $\mathbf{9 0 , 0}$ & 91,4 & $\mathbf{8 3 , 1}$ & $\mathbf{9 4 , 6}$ & 90,5 & 83,0 \\
bj_2 & 94,5 & 86,7 & $\mathbf{9 3 , 1}$ & 82,0 & 91,1 & 92,4 & 82,3 \\
oe_1 & 93,4 & 86,6 & 89,7 & 82,8 & 94,3 & 88,0 & 82,2 \\
bj_3 & 93,1 & 82,7 & 92,1 & 78,3 & 92,8 & 93,2 & 82,2 \\
bj_4 & 92,7 & 88,5 & 89,4 & 82,2 & 91,5 & 89,9 & 81,7 \\
oe_2 & 92,1 & 86,9 & 86,6 & 75,6 & 84,3 & 76,7 & 75,7 \\
oe_3 & 91,9 & 85,9 & 82,4 & 74,7 & 84,3 & 76,2 & 75,1 \\
bj_5 & 91,4 & 88,6 & 92,6 & 81,9 & 93,2 & 93,6 & $\mathbf{8 3 , 1}$ \\
oe_4 & 90,6 & 88,3 & 87,1 & 74,4 & 84,2 & 73,3 & 72,5 \\
bj_6 & 89,4 & 85,9 & 92,6 & 82,6 & 92,1 & 91,7 & 82,9 \\
oe_5 & 88,7 & 82,2 & 82,2 & 73,3 & 82,7 & 74,7 & 74,4 \\
bj_7 & 88,0 & 84,2 & 92,0 & 81,4 & 91,7 & $\mathbf{9 4 , 4}$ & 83,0 \\
tf_2p1z & 87,0 & 79,4 & 84,0 & 66,2 & 81,8 & 82,3 & 78,6 \\
oe_6 & 86,8 & 76,3 & 79,6 & 69,7 & 85,3 & 71,4 & 72,5 \\
tf_2p & 86,5 & 79,3 & 85,4 & 67,9 & 82,6 & 82,4 & 78,8 \\
tf_1p & 64,5 & 62,1 & 75,0 & 68,8 & 88,7 & 84,3 & 79,4 \\
arx1 & 14,3 & 21,4 & 10,6 & 6,9 & 9,2 & 13,9 & 10,6 \\
arx2 & 11,9 & 18,2 & 9,1 & 5,4 & 7,1 & 11,4 & 8,4 \\
\hline
\end{tabular}

O modelo bj_1 apresentou maior $\mathrm{R}^{2}$ para 4 dos 7 casos
(Caso $1,2,4$ e 5), enquanto os modelos $b j \_2, b j \_5$ e $b j \_7$ apresentaram maior $\mathrm{R}^{2}$ para os Casos 3, 7 e 6, respectivamente. As topologias de modelos apresentaram resultados, em ordem decrescente de $\mathrm{R}^{2}$, nesta sequência: modelos BJ, OE, FT e ARX.

O modelo bj_l apresentou o maior coeficiente de determinação médio com $89,7 \%$, seguido dos modelos $b j \_5$, bj_2 e oe_l com $89,2 \%, 88,9 \%$ e $88,1 \%$, respectivamente. Grande parte dos modelos possuem um bom ajuste para a maioria dos 7 casos de validação (acima de 80\%), o que leva à conclusões precipitadas sobre suas qualidades. Para buscar selecionar os melhores dentre aqueles com elevado valor de $\mathrm{R}^{2}$, uma análise de resíduos é proposta, conforme descrito na Seção 3.6 .

Foi observado que vários modelos da Tabela 3 estão polarizados, pois não respeitam o intervalo definido em $10 \%$. A Figura 4 apresenta apenas os modelos que satisfazem as Equações 15 e 16, isto é, possuem curvas de correlação entre os limites definidos. É possível observar que os 7 modelos BJ atenderam as especificações (bj_1 à bj_7), enquanto todos os demais modelos (OE, ARX e FT) não passaram no teste de análise de resíduos.

Estas análises foram feitas para o primeiro conjunto de dados de validação (Caso 1). O comportamento dos resíduos para os demais casos foram analisadas e apresentaram comportamento similares à este Caso.

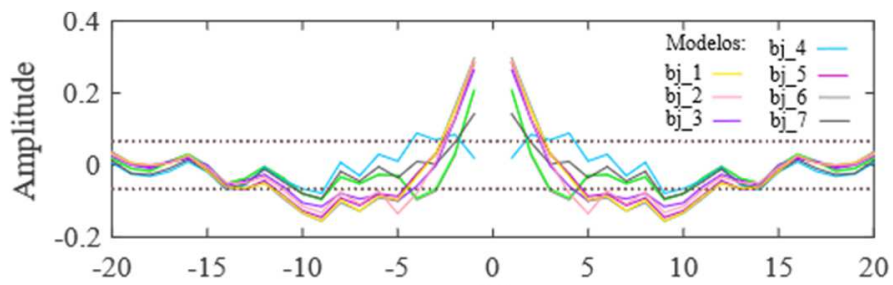

(a)

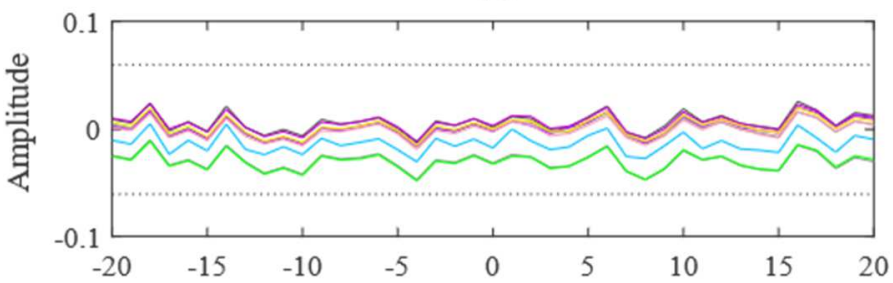

(b)

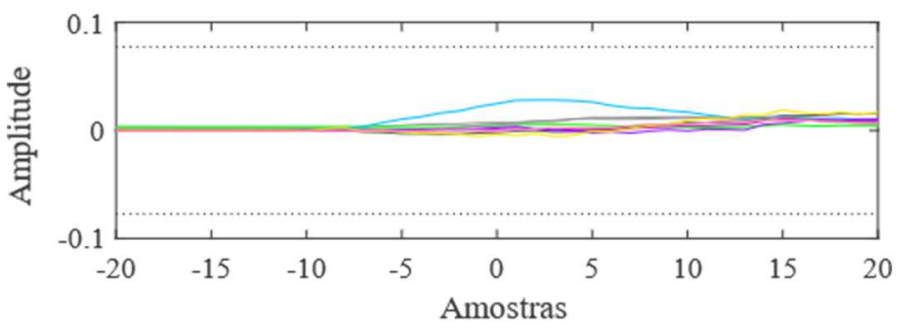

(c)

Figura 4 - (a) Autocorrelação dos resíduos. Correlação entre (b) entrada (vazão) e resíduos e (c) entrada (potência) e resíduos.

4.3 Escolha do modelo e comparação com o modelo caixa 


\section{branca}

Dos 18 modelos propostos, apenas 7 apresentaram resultados compatíveis aos critérios até então discutidos.

Como bj_1 apresentou maior $\mathrm{R}^{2}$ em 4 dos 7 casos, satisfez as análises de resíduos e foi aquele que apresentou o maior coeficiente de determinação médio $(89,7 \%)$, torna-se um ótimo candidato a ser escolhido para comparações com o modelo teórico da Equação 13.

O modelo bj_1 possui 6 parâmetros estimados para o sinal de comando 4-20 mA e 4 parâmetros para a vazão de entrada, conforme apresentado nas Equações 17 à 23:

$$
y_{i}(k)=\frac{B_{i}(z)}{F_{i}(z)} u_{i}(k)+\frac{C(z)}{D(z)} v(k), \text { com } i=\{1,2\},
$$

onde o subíndice 1 indica modelo para a entrada $u_{1}$ (sinal de comando 4-20mA). Portanto, os polinômios $B_{1}(z)$ e $F_{1}(z)$ são dados por:

$$
\begin{aligned}
B_{1}(z)=-4,15 & 10^{-4} z^{-1}+6,4710^{-5} z^{-2} \\
& +3,3110^{-5} z^{-3}-1,2810^{-4} z^{-4} \\
& +5,9510^{-5} z^{-5}+1,5810^{-4} z^{-6},
\end{aligned}
$$

e,

$$
\begin{aligned}
F_{1}(z)=1-0,39 z^{-1}-0,86 z^{-2}+0,56 z^{-3} \\
-0,87 z^{-5}-0,415 z^{-5}+0,97 z^{-6} .
\end{aligned}
$$

O subíndice 2 indica o modelo para a entrada $u_{2}$ (vazão):

$$
\begin{aligned}
B_{2}(z)=-1,4610^{-4} z^{-1}+7,2810^{-6} z^{-2} \\
+8,50510^{-6} z^{-3}-1,710^{-5} z^{-4},
\end{aligned}
$$

e,

$$
\begin{gathered}
F_{2}(z)=1-0,97 z^{-1}+0,05 z^{-2}-0,88 z^{-3} \\
+0,819 z^{-4} .
\end{gathered}
$$

Os polinômios $C(z)$ e $D(z)$ são comuns:

$$
\begin{gathered}
C(z)=1+0,47 z^{-1}-0,32 z^{-2}-0,63 z^{-3} \\
+0,275 z^{-4} \\
\begin{array}{c}
D(z)=1-0,41 z^{-1}-z^{-2}-0,5122 z^{-3} \\
+0,9187 z^{-4} .
\end{array}
\end{gathered}
$$

Uma análise de predições 1 passo à frente, 5 passos à frente e livre pode ser vista na Tabela 4 para todos os Casos. O $\mathrm{R}^{2}$ diminui de $98,98 \%$ para $95,33 \%$ no Caso 1 .

Tabela 4 - Modelo $b j$ _1: Predição 1 passo, 5 passos à frente e predição livre.

\begin{tabular}{llll} 
Casos & \multicolumn{3}{c|}{ Modelo $\boldsymbol{b j}_{\mathbf{1}} \mathbf{1}: \mathbf{R}^{\mathbf{2}}$} \\
& $\mathbf{1}$ passo (\%) & $\mathbf{5}$ passos (\%) & Livre (\%) \\
Caso1 & 98,98 & 97,06 & 95,33 \\
Caso 2 & 98,14 & 95,03 & 90,0 \\
Caso 3 & 98,24 & 94,96 & 91,4 \\
Caso 4 & 96,12 & 87,98 & 83,1 \\
Caso 5 & 98,74 & 96,79 & 94,6 \\
Caso 6 & 98,67 & 96,44 & 90,5 \\
Caso 7 & 95,66 & 86,13 & 83,0 \\
Média & 97,79 & 93,48 & 89,70 \\
\hline
\end{tabular}

Além disso, o $\mathrm{R}^{2}$ médio da predição 1 passo à frente reduz de $97,79 \%$ para $89,70 \%$ quando a predição livre é considerada.

A fim de comparar os resultados obtidos do modelo $b j \_1$ com o modelo proposto por MORAES et al. (2017), a FT da Equação 13 foi simulada para os 7 casos, conforme apresentado na Tabela 5. É possível notar que em todos os 7 casos analisados, o modelo bj_l apresentou melhores resultados de predição, em relação ao modelo caixa branca.

As Figuras 5 (a), (b) e (c) detalha a predição livre dos modelos $b j \_1$ e de MORAES et al. (2017) nos Casos 1, 3 e 5, respectivamente.

Tabela 5 - Comparação $\mathbf{R}^{2}$ e RMSE do modelo proposto e do modelo teórico.

\begin{tabular}{lllll|} 
Casos & \multicolumn{2}{c}{ bj_1 } & \multicolumn{2}{c}{ MORAES et al. } \\
& $\mathbf{R}^{\mathbf{2}}(\boldsymbol{\%})$ & $\mathbf{R M S E}$ & $\mathbf{R}^{\mathbf{2}}(\boldsymbol{\%})$ & $\mathbf{R M S E}$ \\
Caso1 & 95,3 & 0,0467 & 90,45 & 0,0955 \\
Caso 2 & 90,0 & 0,010 & 85,78 & 0,1422 \\
Caso 3 & 91,4 & 0,086 & 65,83 & 0,3417 \\
Caso 4 & 83,1 & 0,169 & 78,19 & 0,2181 \\
Caso 5 & 94,6 & 0,054 & 85,35 & 0,1465 \\
Caso 6 & 90,5 & 0,095 & 80,33 & 0,1967 \\
Caso 7 & 83,0 & 0,170 & 73,59 & 0,2641 \\
Média & 89,7 & 0,090 & 79,93 & 0,2000 \\
\hline
\end{tabular}
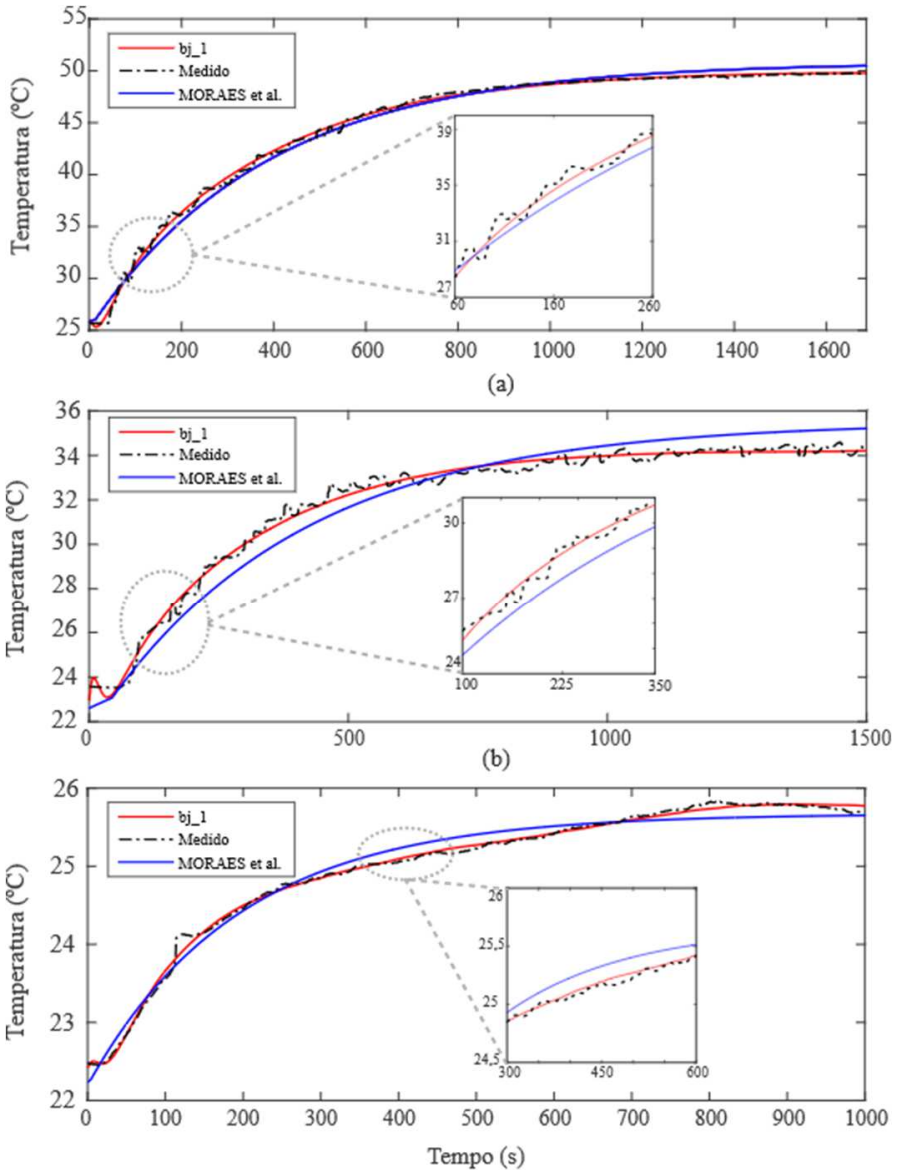

(c)

Figura 5 - Predição do modelo caixa branca para (a) Caso 1. (b) Caso 3. (c) Caso 5.

Algumas observações podem ser retiradas da Tabela 5 e Figura 5. Por exemplo, para o Caso 3: o modelo $b j \_1$ apresenta RMSE $=0,086$ e $\mathrm{R}^{2}=91,4 \%$ enquanto o modelo caixa branca possui $\mathrm{RMSE}=0,3417$ e $\mathrm{R}^{2}=65,83 \%$. Neste exemplo 
específico, há uma grande diferença $(25,57 \%)$ do valor do $\mathrm{R}^{2}$.

Para o Caso 1, o modelo bj_1 apresentou RMSE = 0,046 e $\mathrm{R}^{2}=95,22 \%$ enquanto o modelo caixa branca apresentou $\mathrm{RMSE}=0,0955$ e $\mathrm{R}^{2}=90,45 \%$. A diferença do valor de $\mathrm{R}^{2}$ foi reduzida em relação ao Caso 3, no entanto ainda é de $4,77 \%$.

Finalmente, para o Caso 5, o modelo $b j \_1$ apresentou RMSE $=0,054$ e $\mathrm{R}^{2}=94,6 \%$ enquanto o modelo de MORAES et al. (2017) apresentou $\mathrm{RMSE}=0,1465$ e $\mathrm{R}^{2}=85,35 \%$. A diferença do valor de $\mathrm{R}^{2}$ foi de $9,25 \%$ e ficou próxima à diferença entre as médias, cujo resultado foi de $9,77 \%$.

As análises são similares para os demais casos. Deve estar claro que não foi necessário conhecer a fundo o sistema para encontrar um modelo que o descreve melhor, em relação àquele obtido por meio de aproximações da física do processo.

\section{CONCLUSÕES}

Diferentes modelos para o tanque de aquecimento da planta didática SMAR PD3 foram obtidos neste trabalho. A avaliação e seleção dos melhores modelos foram baseadas em ferramentas como: RMSE, $\mathrm{R}^{\mathbf{2}}$ para predições livres e análise de resíduos. Todas as inferências sob os modelos obtidos foram feitas através de 7 conjuntos de dados de validação (chamados de Casos). Foi selecionado o melhor modelo dos 18 propostos e comparado com o modelo caixa branca disposto em MORAES et al. (2017). A comparação revelou melhores resultados de ajuste para predição livre em todos os casos estudados, com $\mathrm{R}^{2}$ médio maior em $9,77 \%$.

Por fim, o trabalho proporcionou um estudo e a aplicação de ferramentas de identificação e modelagem de sistemas. Foram estudados vários modelos discretos e contínuos, tais como ARX, BJ, OE e FT, comparando-os entre si e selecionando o melhor. De todos os modelos estimados, aquele que apresentou melhor predição livre foi o modelo de $b j \_1$, com 6 e 4 coeficientes para B(z) (duas entradas), 6 e 4 coeficientes para $\mathrm{F}(\mathrm{z})$ e 4 coeficientes para $\mathrm{C}(\mathrm{z})$ e $\mathrm{D}(\mathrm{z})$, apresentando um $\mathrm{R}^{2}$ médio de aproximadamente $89,7 \%$. Foi evidenciado a importância do estudo das técnicas de modelagem apresentadas, assim como as análises de qualidade de modelos, uma vez que poupa tempo do profissional de modelagem aliado à bons resultados práticos.

\section{R E F E R E N C I A S}

AGUIRRE, L. A. Introdução à Identificação de Sistemas. $3^{\text {rd }}$ ed., Minas Gerais: Editora UFMG, 2007.

ANDRADE, Alvaro Batista de; ARAÚJO, Diego Basilio. Desenvolvimento de um simulador para planta didática PD3 da SMAR utilizando estratégia de controle de feedback e cascata. Monografia - Curso de Controle e Automação, Instituto Federal Fluminense, Campos dos Goytacazes, RJ, 2013.
ARRUDA, Gustavo H. M.; BARROS, Péricles R. Estimação da função de sensibilidade baseada em experimento com relé em malha fechada. Sba Controle \& Automação, Campinas, SP, vol.14, n.3, p.235-244, 2013.

CORRÊA, M. V.; AGUIRRE, L. A. Identificação não-linear caixa-cinza: uma revisão e novos resultados. Sba Controle \& Automação, Campinas, SP, v. 15, n. 2, p. 109-126, abr./jun. 2004.

COUTINHO, E. R; SILVA, R. M; DELGADO, A. R. Utilização de Técnicas de Inteligência Computacional na Predição de Dados Meteorológicos. Revista Brasileira de Meteorologia, v. 31, n. 1, p. 24-36, 2016.

DOMINGOS, N. B.; MORAES, C. R.; NICACIO, J. V.; TÔRRES, A. G. Planta didática SMAR pd3: ajuste dos parâmetros do controlador PI do tanque de aquecimento - parte B. The Journal of Engineering and Exact Sciences - JCEC, Viçosa, MG, v. 03, n. 3, p. 476-498, 2017.

GOMES, N. C.; NICACIO, J. V.; TÔRRES, A. G. Planta didática SMAR pd3: simulação e ajuste dos parâmetros do controlador de temperatura do tanque de mistura. The Journal of Engineering and Exact Sciences - JCEC, Viçosa, MG, v. 03, n. 7, p. 933-954, 2017.

JOHANN, G.; PEREIRA, N; SILVA, E. Avaliação de modelo matemático de parâmetros distribuídos aplicado à secagem de grãos de uva em camada fina. XXXVII Congresso Brasileiro de Sistemas Particulados, v.2, n. 1, p. 521-530, out. 2015, São Paulo.

KLUEVER, C. A. Sistemas dinâmicos: modelagem, simulação e controle. $1^{\text {st }}$ ed., Rio de Janeiro: LTC, 2017.

MARQUES, I. S. Predição de Séries Temporais Utilizando Algoritmos Genéricos. 66 f. Tese (Mestrado em Ciência da Computação) - Universidade Federal do Rio Grande do Sul, Porto Alegre, RS, out. 2012.

MORAES, C. R.; DOMINGOS, N. B.; NICACIO, J. V.; TÔRRES, A. G. Planta didática SMAR pd3: modelagem e simulação do tanque de aquecimento - parte A. The Journal of Engineering and Exact Sciences - JCEC, Viçosa, MG, v. 03, n. 3, p. 454-475, 2017.

OLIVEIRA, L. M. et al. Utilização de uma planta didática smar para complementação do ensino de engenharia de controle e automação. In: Congresso Brasileiro de Educação em Engenharia, Belém, PA, set. 2012.

SANTOS, T. M. Técnicas de Realimentação Aplicadas na Planta Didática SMAR PD3 Foundation Fieldbus. $124 \mathrm{f}$. Monografia - Curso de Engenharia Elétrica, Universidade Federal de Ouro Preto, João Monlevade, 2016.

SMAR. Manual de instruções: Operação e manutenção - plantas didáticas. Maio, 2012. Disponível em: < http://www.smar.com/brasil/produto/pd3-plantadidatica-hart-foundation-fieldbus-e-profibus>. Acesso em 6 de julho, 2018.

WERNER, L; RIBEIRO, J. L. Previsão de demanda: uma aplicação dos modelos Box Jenkins na área de assistência técnica de computadores pessoais. Gestão \& Produção, v. 10 , n. 1 , p. 47-67, abr. 2003. 